\title{
Leviathan 3/2010
}

\section{Dagmar Simon}

\section{Zu diesem Heft}

Die Art und Weise des wissenschaftlichen Publizierens ist „,ins Gerede geraten“. Einerseits spielt in den allfälligen Evaluationen von Personen, Institutionen und in der Forschungsförderung das Zählen von Publikationen als Bewertungsgrundlage eine immer größere Rolle. Andererseits wird im Zuge der Open Access-Diskussion heftig darüber debattiert, wem eigentlich der veröffentlichte Aufsatz gehört, wie die Zugangsrechte der Rezipienten aussehen und vieles mehr. Grund genug, sich als sozialwissenschaftliche Zeitschrift diesem Thema mit mehreren Beiträgen zu widmen: sowohl aus der Perspektive betroffener Wissenschaftler/innen als auch der der Bibliotheken, die sich in einem rasanten Tempo auf neu entstehende Märkte einzustellen haben.

Alfred Kieser befasst sich mit den Auswirkungen einer Quantifizierung von Forschungsleistungen und den Vorgaben von Anreizen zu ihrer Steigerung, welche die intrinsische Motivation der Wissenschaftler/innen zunehmend verdrängt.

Seine radikalen Lösungsvorschläge: das System der leistungsabhängigen Zulagen für Ergebnisse in Forschung und Lehre abschaffen, auf den sich aus intrinsischer Motivation speisenden Wettbewerb zwischen den Institutionen setzen und Evaluationen nur noch in begründeten Ausnahmefällen initiieren.

Die beabsichtigten und unbeabsichtigten Folgen der Publikationsstrukturen wie bspw. das höhere Gewicht von Zeitschriftenveröffentlichungen durch die Zitatdatenbanken gegenüber Büchern und Buchbeiträgen und die Bevorzugung von Forschung, die kurzfristig veröffentlichungsfähige Resultate erwarten lässt im Vergleich zu langfristig angelegten Programmen mit ungewissen Ausgang, thematisiert Manfred Boni. Er zeigt auch, dass der Publikationsmarkt durch verfestigte, asymmetrische Strukturen geprägt ist, die den großen Wissenschaftsverlagen eine nahezu monopolartige Stellung verschaffen und die auch Open Access bislang nicht aufbrechen konnte.

Wenn etwas Open Access gestellt wird, ist es für jedermann kostenfrei zugänglich und für jeden verantwortlichen Zweck nutzbar. Rainer Kuhlen diskutiert diesen Wechsel unter dem Begriff der Gemeingüter bzw. der Wissensallmende diskutiert. Sein Resümee: Open Access ist ein wichtiger Baustein in der Wissenschaftskommunikation, „da weder Marktmechanismen noch staatliche Regulierungsformen wie das Urheberrecht dem offenen und freizügigen Austausch von publiziertem Wissen Rechnung trage, der für Bildung und Wissenschaft konstitutiv ist und damit im Interesse der gesamten Gesellschaft liegt".

(C) VS Verlag für Sozialwissenschaften 2010 
Klaus-Rainer Brintzinger sieht Open Access nicht nur als Modell des freien Zugangs zu wissenschaftlichen Texten, sondern auch als Möglichkeit, die durch die astronomischen Preise für Zeitschriftenabonnements ausgelöste Krise für sehr begrenzte Bibliotheksetats zu lösen. Und es verändert die Bibliotheken grundsätzlich: Mit den von ihnen betriebenen Repositorien beteiligen sie sich erstmals seit dem Ende der mittelalterlichen Kloster-Skriptorien wieder selbst am Produktionsprozess. Das World Wide Web steigert die Verfügbarkeit von Informationen weltweit, potenziert aber auch die Unüberübersichtlichkeit und stellt damit vor allem Archivinstitutionen vor neue Herausforderungen. Roland S. Kamzelak plädiert dafür, das Internet durch die Redaktion von Inhalten und die Aufbereitung von Metadaten verlässlicher zu machen. Bei den Nutzern kann somit ein „Grad an Bewusstsein für Qualität geschaffen (werden), der in der, Tintenwelt‘ selbstverständlich sei“.

Die Beobachtung, wie sich Open Access auf das Publikationsverhalten von Wissenschaftlern auswirkt, das nicht von monetären Anreizen sondern durch das Reputationssystem geprägt ist, wird weiterhin spannend werden; auch ob sich hierdurch die Strukturen und Mechanismen des Publikationsmarkts weiter verändern. Im Hinblick auf die vielfach kritisierten Auswirkungen von Evaluationsverfahren ist darauf zu achten, dass das Kind nicht mit dem Bade ausgeschüttet wird. Denn auf Problemlagen des deutschen Wissenschaftssytems im ,,vorevaluativen Zeitalter“, bspw. in der Nachwuchsförderung oder der Gefahr der Versäulung in der Forschungslandschaft, haben dann Evaluationen die Aufmerksamkeit gelenkt und eine produktive Debatte ausgelöst. Und auch beim Zählen und Messen ist ein wenig Land in Sicht: Die Deutsche Forschungsgemeinschaft, die VolkswagenStiftung und auch die amerikanische National Science Foundation haben die Anzahl der Publikationen, die für Förderanträge aufgeführt werden müssen, radikal begrenzt. Der DFG-Präsident Matthias Kleiner kritisiert, dass ,die numerischen Kriterien zur Leistungsbeurteilung eines Forschers die Vielschreiberei begünstigt“" hätten. Sicherlich gilt für Evaluationen generell, dass weniger mehr ist.

$\mathrm{Zu}$ den weiteren Beiträgen dieses Hefts: Michael Hartmann befasst sich mit den Folgen einer durch die Exzellenzinitiative eingeleiteten vertikalen Ausdifferenzierung der Hochschullandschaft und der Konzentration der Ressourcen. Der Nationalstaat ist unter den Perspektiven von Globalisierung, Europäisierung und Privatisierung Thema von drei Artikeln: Unter dem Titel „Zivilisierung der Weltordnung“ beschäftigen sich Cord Jakobeit, Robert Kappel und Ulrich Mückenberger mit der Rolle der Normbildung und -durchsetzung durch transnationale Netzwerke, gegenüber denen staatliche Akteure an Boden verlieren. Die Rolle des Nationalstaats analysiert Claudio Franzius im Lichte des Lissabon-Urteils des Bundesverfassungsgerichts, Nicole Deitelhoff und Anna Geis fragen, ob sich der Leviathan entkernt und analysieren die funktionelle Umrüstung in der Sicherheits- und Verteidigungspolitik westlicher Demokratien. 